\title{
Predictive value of image cytometry for diagnosis of lung cancer in heavy smokers
}

\author{
S. Xing*, B. Khanavkar*, J.A. Nakhosteen*, Z. Atay", K-H. Jöckel" and W. Marek*, \\ for the Research Institute for Diagnosis and Treatment of Early Lung Cancer \\ (RIDTELC) Lung Study Group
}

ABSTRACT: The Research Institute for Diagnosis and Treatment of Early Lung Cancer (RIDTELC) Lung Study was initiated to determine whether lung cancer screening by automated sputum cytometry combined with conventional sputum cytology and auto-fluorescence in addition to white light bronchoscopy could enhance the detection rate of early lung cancer. The present study analyses the initial findings to evaluate the efficiency of automated sputum cytology in predicting the diagnosis of lung cancer.

In this study, malignancy grade was used as a predictive parameter for lung cancer. In total, 2,480 heavy smokers ( $>30$ pack-yrs), aged 50-74 yrs, with no previous cancer in the last 5 yrs, received chest radiology, conventional sputum cytology and sputum cytometry screening.

In total, twenty-seven lung cancers were diagnosed, representing a prevalence of 1.1\%, 25 of which provided sputum samples. Positive automated sputum cytology results were seen in 176 smokers $(7.2 \%), 10(0.4 \%)$ of whom had severe dysplasia or higher lesions (positive results) by conventional sputum cytology examination. Out of 25 tumour cases, 20 had suspicious results using automated sputum cytology, representing $80 \%$ sensitivity. One patient out of 24 with tumours had positive results on conventional sputum cytology, representing a sensitivity of $4.2 \%$. For all stages of squamous cell lung cancer and later stage adenocarcinoma the sensitivity of automated sputum cytology was $100 \%$. For adenocarcinoma stage I sensitivity was $25 \%$.

In conclusion, DNA analysis of sputum slides by automated sputum cytology may be a suitable tool for the detection of early lung cancer and the characterisation of a high-risk group with preinvasive lesions for follow-up.

KEYWORDS: Automated image cytometry, DNA analysis, early lung cancer screening, heavy smokers, sputum cytology

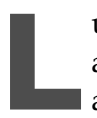

ung cancer is one of the most prevalent and lethal cancers, accounting for $17.8 \%$ of all cancer deaths [1]. Since the 1970s, the 5yr survival rate for lung cancer has remained unchanged at $<15 \%$ [2]. It has been recognised that the prognosis of lung cancer is strongly associated with the stage of cancer at the time of diagnosis, and 5-yr survival rates range from 5\% for patients with stage IV lesions to $80 \%$ for patients with stage I [3]. Therefore, improving the detection rate of early-stage lung cancer is essential for improving the prognosis of lung cancer. Currently, sputum cytology is the most commonly used noninvasive method for early diagnosis. Although the value of conventional cytology is undisputed, data from three large studies [4-6], conducted in the 1970s in the USA, have suggested that conventional sputum cytology is not suitable for mass screening due to its poor sensitivity. However, sputum is considered to be promising material for the early recognition of cancer present in the airways. All histological types of lung cancer are detectable in sputum with modern methods such as DNA and automated image cytometry [7]. To improve the sensitivity of sputum examination as a screening tool for detection of early lung cancer, a variety of molecular biological and other parameters have been proposed, such as markers for cell proliferation, oncogene amplification and growth factor overexpression. Several other approaches are being developed, e.g. immunostaining, PCR techniques and computer-assisted image analysis [8].

Computer-assisted image analysis (automated image cytometry) represents a new, sensitive method for the quantitative analysis of the nuclear structure and DNA content of individual cells [9]. The automated image cytometry used in
AFFILIATIONS

*Augusta Teaching Hospital, Bochum,

\#Institute for Cytology, Hannover, and

"Institute for Medical Informatics, Biometry and Epidemiology, University Clinics of Essen, Essen, Germany.

CORRESPONDENCE

W. Marek

Research Institute for Diagnosis and Treatment of Early Lung Cancer (RIDTELC)

Augusta Teaching Hospital

Bergstr. 26

D-44971 Bochum

Germany

Fax: 492345172463

E-mail: Wolfgang.Marek@

ruhr-uni-bochum.de

Members of the RIDTELC study group include: J.A. Nakhosteen,

B. Khanavkar, W. Marek, A. Scherff, A. Muti, M. Abdul-Wahed, S. Peters, S. Philippou and R. Heikemann (Bochum, Germany); P. Farschou (Copenhagen, Denmark); K-H.

Jöckel, J. Hüsung (Essen, Germany); Z. Atay and Th. Topalidis (Hannover, Germany).

Received:

October 232003

Accepted after revision:

February 012005

European Respiratory Journal

Print ISSN 0903-1936

Online ISSN 1399-3003 
this study is based on the measurement of DNA content of individual exfoliated epithelial cells in the airways. By cytometric evaluation of nuclear DNA content, a DNA stem line in an abnormal histogram position is often termed a "DNA aneuploidy". This is assumed to be the cytometric equivalent of chromosomal aneuploidy [10], which has been thought to be a reliable marker of cancer [11]. Several studies have demonstrated its advantage over flow cytometry in measuring DNA content in cells on cytological routine slides [12]. In several studies, image cytometry has been used to quantify the DNA content of cells, with the intention to differentiate malignant from benign lesions in various tissues, e.g. lung, oral, mucosal cervix, bladder and others [13-15].

In view of the vital importance of early detection of lung cancer and the advent of new technologies, such as automated image cytometry and auto-fluorescence bronchoscopy, a populationbased prospective single-blind, controlled clinical trial was initiated in May 2000 in the Research Institute for Diagnosis and Treatment of Early Lung Cancer (RIDTELC; Bochum, Germany), in order to examine the efficiency of these techniques in improving the detection rate of early lung cancer. The current study presents the findings of the initial examination and uses them to evaluate the predictive value of DNA analysis by image cytometry in the diagnosis of lung cancer.

\section{MATERIALS AND METHODS Study population}

In total, 2,889 smokers interested in the RIDTELC Lung Study in the period from May 2000 to April 2002 contacted the study administrator by telephone after announcement of the study in the local newspapers and on local radio stations. Heavy smokers with a cumulative cigarette consumption of $\geqslant 30$ pack-yrs aged 50-74 yrs were eligible for the study. After excluding individuals either out of the age and exposition range or with a history of cancer in the last $5 \mathrm{yrs}$, a total of 2,480 voluntary suitable participants were recruited from the general population in the Ruhr industrial area around Bochum $(n=2,422)$ and a smaller group from the urban region of Copenhagen (Denmark) $(n=58)$. After giving written informed consent, each participant completed a questionnaire providing demographic data, pertinent medical history, smoking history, socio-economic information and occupational exposure history to harmful agents. The list of occupational exposures, adopted from JÖCKEL et al. [16], included 28 occupational groups and asked for educational levels. Special emphasis was given to asbestos exposure and working in the mining industry.

After lung function testing, sputum was collected following induction with hypertonic saline solution, and a digital chest radiograph investigation was performed. All data were collected by an independent study monitor and archived on a password-protected PC with access for the study monitor only. The study design was approved by the ethics committee of the Augusta Teaching Hospital (Bochum, Germany).

\section{Chest radiograph screening}

All participants had posterior-anterior and lateral digital chest roentgenograms (Thoravision with Selen-detector-head, super $50 \mathrm{CPD}$ generator, $50 \mathrm{~kW}, 150 \mathrm{kV}$, frame $43 \times 49 \mathrm{~cm}$; Phillips, Aachen, Germany), which were read by a radiologist. The results of these radiographs were interpreted as: 1) no abnormality detected; 2) abnormalities not suggestive of malignant tumour; or 3) suspected malignant tumour. All abnormal chest films were re-examined by a pulmonologist. All participants with suspected malignant tumours were called for computer tomography (CT) examination.

\section{Sputum collection}

Under the supervision of a respiratory therapist, all participants underwent sputum induction by inhalation of aerosolised 3\% saline with the addition of salbutamol (25 mg into $20 \mathrm{~mL}$ saline) for $20 \mathrm{~min}$ using an ultrasonic nebuliser (Aneasthoschall 880; HICO, Cologne, Germany) or a jet nebuliser (Pari Sole, Starnberg, Germany). A group of 67 $(0.3 \%)$ participants who did not produce sufficient sputum after induction were asked to collect additional spontaneous sputum on the following 3 days. Sputum was collected in 50$\mathrm{mL}$ labelled centrifuge tubes, filled with $25 \mathrm{~mL}$ of saccomanno preservative, with the addition of $0.1 \%$ dithiothreitol (DTT).

\section{Slide preparation}

The liquefied sputum specimens in the saccomanno-DTT solution were centrifuged at $500 \times g$ for $15 \mathrm{~min}$. The supernatant was decanted and the cell pellets were resuspended in 0.5-2.5 mL of saccomanno solution, according to weight. Lastly, two drops of the cell suspension were placed onto each of six slides and smeared with capillary forces using a separate glass slide. Out of the six air-dried slides, two were stained according to the methods of PAPANICOLAOU [17] for conventional cytology, two were stained according to the modified Feulgen reaction for DNA image cytometry [18] and the remaining two were stored for future reference.

\section{Conventional sputum cytology}

Sputum cytology examination and DNA image cytometry were independently performed without the knowledge of clinical results. The cytology slides were sent to the Institute of Cytology (Hannover, Germany) to be screened by cytotechnologists and cytopathologists from the institute. The results, presented according to a modified classification of PAPANicolaOU [19], were sent directly to the study administrator.

\section{DNA image cytometry}

In the present study, image cytometry was automatically carried out using the Cyto-Savant system (Cyto-Savant ${ }^{\circledR}$; Oncometrics, Vancouver, Canada) adopted for sputum specimens [9]. The feulgen-thionin-stained slides were used to quantify nuclear DNA content. After DNA staining, the nuclear-integrated optical density is the cytometric equivalent of its DNA content. The technology was adapted to the recommendations of the European Society for Analytical Cellular Pathology (ESACP) Task Force on standardisation of diagnostic DNA image cytometry [20]. For each slide, almost 2,000 exfoliated cells were collected, which included: 1,000 epithelial cells; up to 200 suspicious nuclei with a DNA-index $>1,125$ and $<2.5$; up to 100 highly suspicious nuclei with a DNA index $>2.5$ times the normal DNA content; $\sim 200$ each of lymphocytes, polymorphonuclear and eosinophil granulocytes; and 100 alveolar macrophages. Before measurement, the cell galleries were reviewed to eliminate the nuclei that 
were blurred or overlapping. The normal $2 \mathrm{c}$ reference value was established by measuring the normal well-preserved lymphocytes in each slide as internal reference cells, whose co-efficient of variation was $<12.5 \%$ (SD/mean). Hence, the DNA content of normal epithelial nuclei, termed as euploidy, was $2 \pm 0.25 c$; a cell was defined as aneuploid if its DNA content deviated from $2 \pm 0.25 \mathrm{c}$ or the integer-valued exponents of $2 \mathrm{c}($ e.g. $4 \pm 0.5 \mathrm{c}, 8 \pm 1 \mathrm{c}, 16 \pm 2 \mathrm{c})$.

Two parameters were applied to describe the DNA content of each cell: the $5 c$ exceeding rate $(5 c E R)$ and the $2 c$ deviation index (2cDI). $5 \mathrm{cER}$ was defined as the percentage of aneuploid cells having a DNA content $>5 \mathrm{c}$. $2 \mathrm{cDI}$ represented the mean squared deviation from the diploid value or the variance around the 2c peak. It was calculated as the sum of squares of the differences between the DNA values of single cells (ci) and the $2 \mathrm{c}$ value, divided by the number of measured cells (n), as follows [21]:

$$
2 \mathrm{cDI}=\sum(\mathrm{ci}-2 \mathrm{c})^{2} \times 1 / \mathrm{n}
$$

A third parameter, the malignancy grade (MG) was calculated from the $2 \mathrm{cDI}$ and $5 \mathrm{cER}$ using a modified formula as follows:

$$
\mathrm{MG}=\log (2 \mathrm{cDI} \times(1+5 \mathrm{cER} \times 10)+1) \times 1.685
$$

This was used to determine the cytometric diagnosis as follows. 1) Grade I: benign, normal nuclear structures, euploidy, MG <0.10; 2) Grade II: suspicious samples with MG $\geqslant 0.10$. 3) Grade 0: nonrepresentative because of inadequate diagnostic cells.

\section{Bronchoscopy and pathology examination}

All patients with severe dysplasia or higher grade changes seen with conventional sputum cytology, or grade II at image cytometry were called for bronchoscopy. Conventional whitelight bronchoscopy (WLB) fluorescence was performed on subjects, followed by light-induced fluorescence endoscopy (LIFE). The findings were reported as normal or suspicious for tumour.

During bronchoscopy, biopsy specimens were taken from all areas suspicious of dysplasia or worse on WLB or LIFE examination, and at least from one side of the normal mucosa. Two to six biopsies were taken from every subject. The histopathological type and grade of diagnosis were determined according to the World Health Organisation (WHO) classification. From the resulting series of biopsies in each case, the most advanced degree of histological change with respect to lung malignancy was used as the final diagnosis in the analysis.

\section{Diagnostic procedure}

At the beginning of the study, all participants underwent spirometry, sputum induction and chest radiographical examination. For participants with a suspicious radiograph, further examinations, such as CT of the chest and bronchoscopy, were performed to obtain a final diagnosis. All patients with severe dysplasia or higher grade changes on cytological examination or grade II with DNA image cytometry were also called for bronchoscopy (conventional and autofluorescence).

\section{Data analysis}

The distribution of continuous variables was examined with respect to skewness and kurtosis. The unpaired t-test, nonparametric test (Kruskal-Wallis or the Mann-Whitney U-test) and Pearson's Chi-squared test were employed to determine the difference of results from various variables between different groups. All statistical tests were two-sided, and statistical significance was determined by a p-value $<0.05$. The receiver operating characteristic (ROC) curves for MG and 5cER were plotted to determine the suitable threshold and to estimate the efficiency of image cytometry relating to the "gold standard" clinically relevant lung cancers, which were defined as histopathologically confirmed severe dysplasia and/or higher grade lesions. The comparison between the areas under the ROC curves for MG and 5cER were made using the method of DELONG et al. [22]. The mean values for 2cDI, 5cER and MG were calculated along with the $95 \%$ confidence intervals (CI) for grade I and II image cytometry results, as well as for histologically classified normal, pre-invasive and invasive biopsies.

\section{RESULTS}

The study population characteristics in terms of DNA image cytometry diagnosis

The demographic and clinical characteristics of the study population are summarised in table 1. In total, 2,480 smokers (71\% males) were included, (mean $\pm \mathrm{SD}$ ) age $59.6 \pm 5.9 \mathrm{yrs}$ and $58.6 \pm 25$ pack-yrs, who started smoking at age $17.7 \pm 3.4$ yrs. The majority of the study population had no history of respiratory disease. Nearly half of the subjects had a family history of cancer and occupational exposure to harmful agents.

Subjects with suspicious cytometric results smoked more heavily than those with normal cytometric results. More subjects in the group with suspicious results had moderate or strong cough and harmful agents exposure history. It is worth mentioning that $>80 \%$ of all subjects with moderate cough had $>1$-yr cough history. For the remaining features listed in the table, there were no statistically significant differences between the groups.

\section{Initial automated sputum cytometry, conventional sputum cytology and chest radiograph screening findings}

Out of 2,480 participants, 2,461 provided adequate sputum for automated sputum cytology and 2,449 for conventional sputum cytology. All 2,480 subjects underwent chest radiographical examination. To date, a total of 27 cancers (representing a prevalence of $1.1 \%$ ) have been found, 20 cancer patients had grade II automated sputum cytology results and one had a positive conventiona sputum cytology result. Lowgrade lesions (metaplasias, and mild and moderate dysplasias) were found in two out of the 24 cancer patients receiving cytological examination (table 2).

In total, 176 subjects had grade II results by image cytometry, of which 20 were diagnosed as cancers, representing a sensitivity of $80 \%$. One tumour was diagnosed in patients with positive cytology, representing a sensitivity of $4.2 \%$. Out of 2,480 participants, 138 (5.6\%) were suspect for lung cancer by chest radiographical examination, of which 17 lung cancers were diagnosed. Out of the 138 subjects with suspect results on chest films, 127 (92\%) underwent a CT examination, 76 were considered to be suspect for malignant lesions by $\mathrm{CT}$, of which 
TABLE 1 Study population characteristics in terms of DNA image and cytometric diagnosis\#

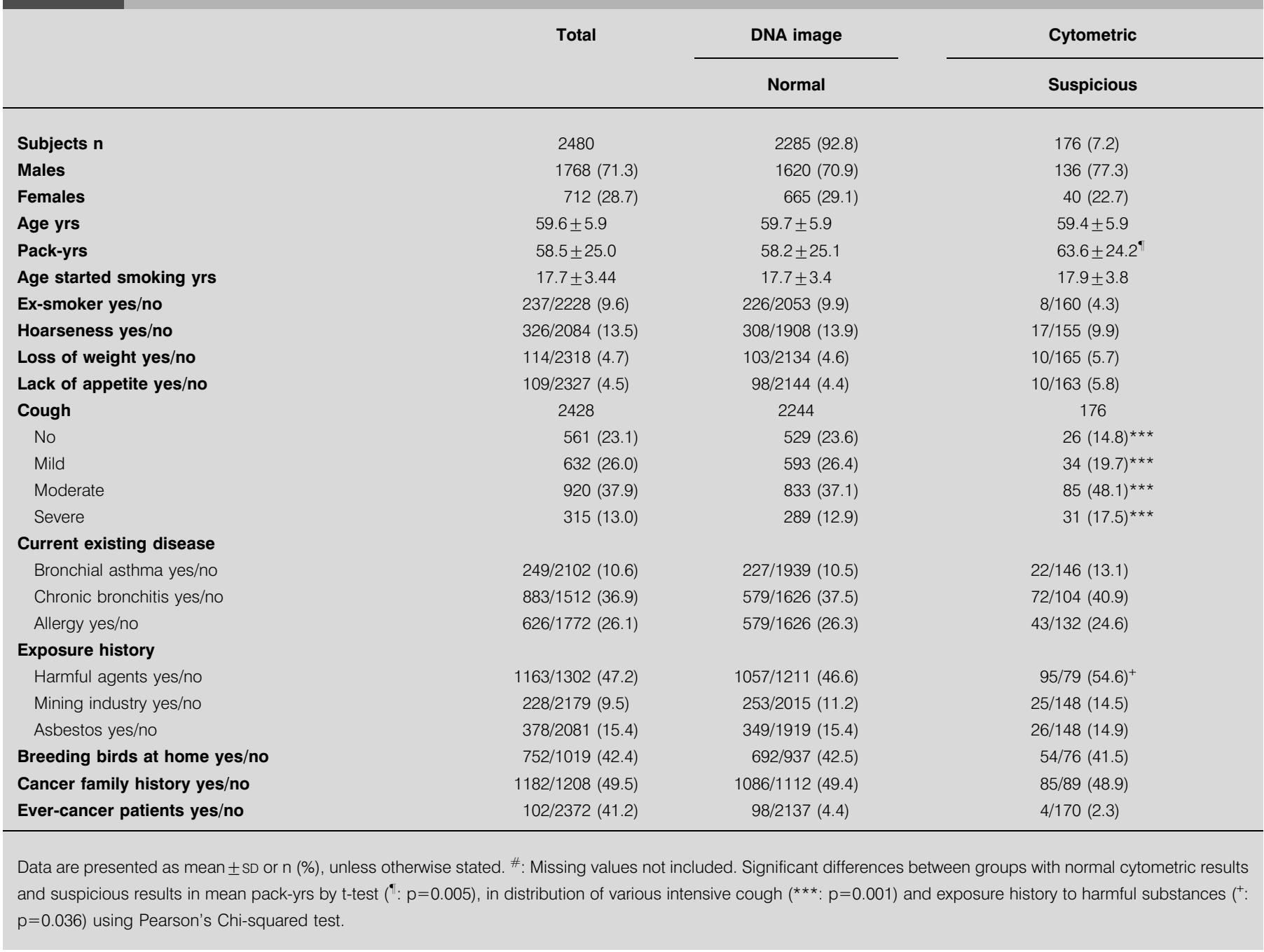

11 were finally diagnosed as cancers, the other six tumours were diagnosed by other diagnostic methods such as a sonography operation.

In total, cancer was detected in 27 patients by screening, 10 patients had both chest radiograph and sputum positive results, 10 had only cytometry suspicious results, one had both cytological and cytometry positive results, and six had only chest radiography suspect results.

\section{Findings of bronchoscopy}

The bronchoscopic findings for tumours were classified as normal or suspect. In total, 95 subjects who had grade II results detected by image cytometry underwent bronchoscopy (out of those six also had positive sputum cytology results). Seven were confirmed as clinically relevant lung cancer by pathological analysis. Out of six participants who had positive results on sputum cytology, one was confirmed by pathological examination. A total of 201 biopsies were taken, of which 49

TABLE 2 Initial findings by sputum image cytometry, conventional cytology examination and radiographical screening

\begin{tabular}{|c|c|c|c|c|c|c|}
\hline & \multicolumn{2}{|c|}{ Cytometry } & \multicolumn{2}{|c|}{ Cytology } & \multicolumn{2}{|c|}{ Chest radiography } \\
\hline & Grade II & Grade I & High grade & Normal/low grade & Suspicious & Nonsuspicious \\
\hline Cancer & $20(11.4)$ & $5(0.2)$ & $1(10)$ & $23(0.9)$ & $17(12.3)$ & $10(0.4)$ \\
\hline No cancer & $156(88.6)$ & 2280 (98.9) & $9(90)$ & 2416 (90.6) & $121(87.7)$ & 2332 (99.6) \\
\hline Total & 176 & 2285 & 10 & 2439 & 138 & 2342 \\
\hline
\end{tabular}

Data are presented as $n(\%)$ 
had suspect results (table 3). No severe dysplasias were diagnosed by pathology in biopsies with normal results. Using the authors' gold standard, there were no false-negative results using the bronchoscopy procedure, taking endoscopically abnormal mucosa and high-grade alterations in histopathology as the cut-off points. From biopsies which were found to be suspect for tumour under bronchoscopy, seven out of 40 patients were diagnosed with clinically relevant lung cancer and one was diagnosed with a benign tumour. The seven patients included three with severe dysplasia, two with carcinoma in situ, one with stage $\mathrm{Ib}$ and one with stage III lung cancer (table 3). Out of the participants, 41 (43.2\%) had lowgrade lesions (27 metaplasias, nine mild dysplasias and five moderate dysplasias).

\section{DNA image cytometric results and histopathological diagnosis}

According to the WHO/International System for Staging Lung Cancer Histological Classification of Lung and Pleural Tumours [3], all histological diagnoses were classified into three main categories: 1) normal (benign); 2) pre-invasive lesions, including squamous dysplasia (mild, moderate and severe) and carcinoma in situ; and 3) malignant or higher grade lesions than carcinoma in situ.

Among the 176 cases with suspicious (grade II) cytometric results, 99 had histological results from biopsy by either bronchoscopy or surgery. The results of $5 \mathrm{cER}$ and $2 \mathrm{cDI}$, as well as MG, of 99 participants with pathological results were plotted against the diagnoses obtained from the histopathological results in figure 1 . There was an association between the different phases in the development of malignancy and the value of $5 \mathrm{cER}$, the median value of which increased from 0.034 to 0.051 and $0.070 \%$ with the progression from histological normal through pre-invasive to invasive tumour changes (Kruskal-Wallis test $\mathrm{p}=0.019$; Mann-Whitney U-test $\mathrm{p}=0.021$ for the group with histological normal and pre-invasive results; and $p=0.023$ for the group with normal and invasive results). The median of MG significantly increased from 0.121 to 0.153 with the progression from histological normal to preinvasive tumour changes (Mann-Whitney U-test $\mathrm{p}=0.004$ ). Nevertheless, a significant association between the progression

\begin{tabular}{lcc}
\hline TABLE 3 & $\begin{array}{l}\text { Findings of bronchoscopy versus } \\
\text { histopathological results }\end{array}$ & \\
\hline Histopathological results & Abnormal & Normal \\
\hline No abnormal findings & 2 & 15 \\
Inflammation & 13 & 102 \\
Metaplasia & 20 & 21 \\
Mild dysplasia & 3 & 11 \\
Moderate dysplasia & 3 & 3 \\
Severe dysplasia & 3 & 0 \\
Carcinoma in situ & 2 & 0 \\
Invasive carcinoma & 2 & 0 \\
Benign tumour & 1 & 0 \\
\hline Data are presented as $\mathrm{n}$. & &
\end{tabular}

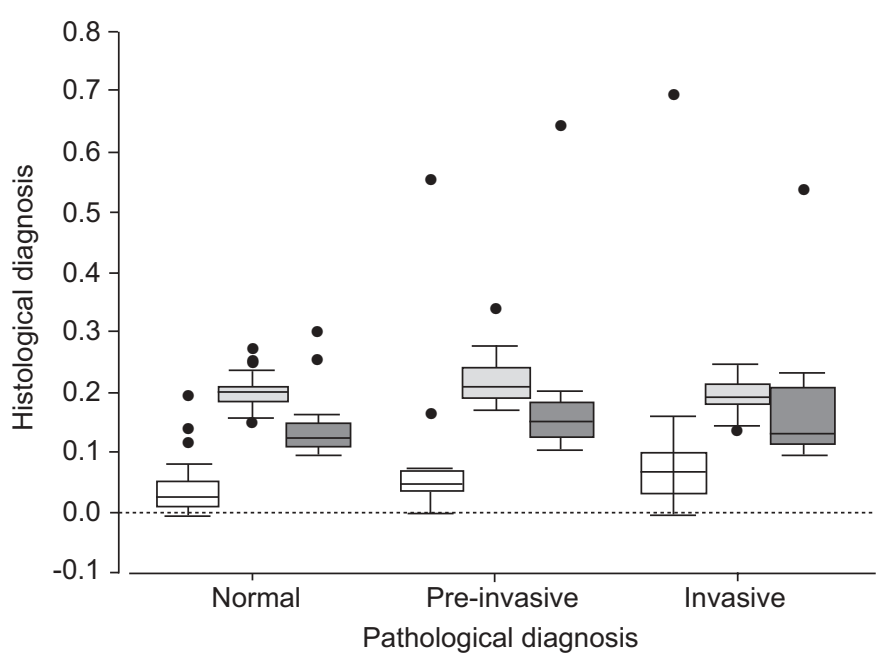

FIGURE 1. Results of $5 c$ exceeding rate $(\square), 2 c$ deviation index ( $\square$ ) and malignancy grade $(\square)$ versus histopathological diagnosis in normal $(n=66)$, preinvasive $(n=17)$ and invasive $(n=16)$ participants. The lower and upper box boundaries represent the 25th and 75 th percentiles. The horizontal lines under and above the box represent the 10th and 90th percentiles, respectively. The median value is shown as the horizontal line within the box. $\mathbf{0}$ : outliers.

of histopathological alterations and 2cDI was not found (Kruskal-Wallis Test $\mathrm{p}=0.21$ ).

\section{ROC curves for MG and 5cER}

To estimate the diagnostic accuracy of automated image cytometry using $\mathrm{MG}$, or $5 \mathrm{cER}$ as decision parameters in predicting lung cancer, the optimum cut-off points were first derived for the two parameters. As the decision cut-off point of the test varied, the sensitivity and specificity of the test also changed. These values are plotted and joined to produce the ROC curve, in which the $\mathrm{Y}$ axis represents sensitivity (the proportion of true-positive results), the $X$ axis represents $1-$ specificity, and the area under the curve indicates the accuracy of the test with different cut-off points. The optimum cut-off levels of MG of 0.10 and 5cER of 0.03 were determined by selecting the point on the ROC curve which maximised both sensitivity and 1-specificity. The accuracy of automated sputum cytology in predicting clinically relevant cancers was $87 \%$ (95\% CI: 77.6-96.5) using MG as the decision parameter and $81.2 \%$ (95\% CI: 69.9-92.5) using 5cER as the decision parameter, which is significantly lower than using MG $(\mathrm{p}<0.05$; fig. 2).

\section{Histological type and stage of lesions found in the study versus DNA image cytometric results}

Out of the 25 patients with cancers detected, one had no cytological results because of inadequate material. The main histological types of the cancers found in this study were adenocarcinoma and squamous cell cancer. All nine patients with squamous cell lung cancer and six with later stage adenocarcinoma had suspicious or grade II cytometric results. Only one out of four with a stage I lesion had suspicious cytometric results. Out of all cancer patients, only two had abnormal results by conventional sputum cytology (table 4). There were four tumours with other histological types which are listed in table 4 , including one undifferentiated carcinoma, 


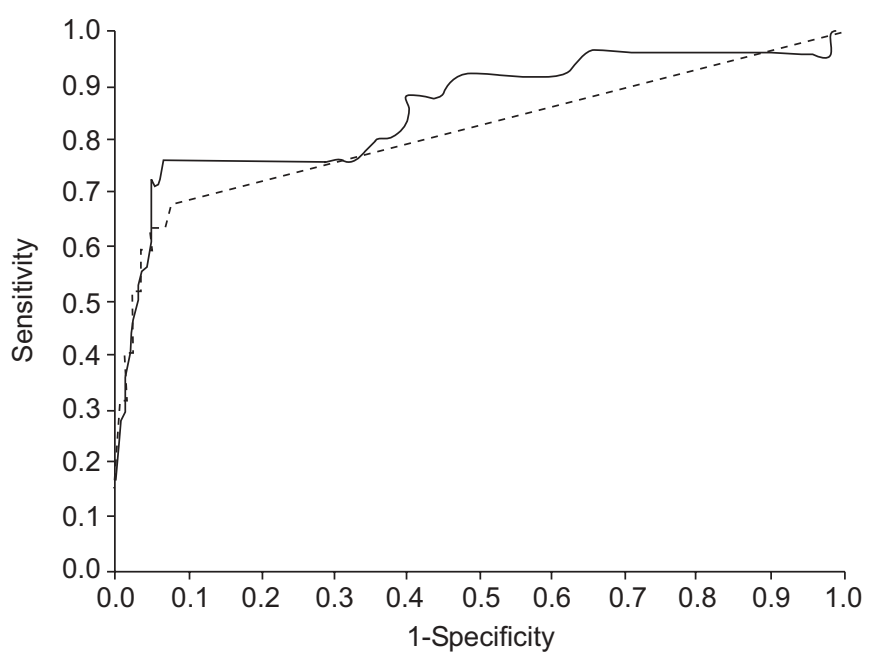

FIGURE 2. Receiver operator characteristics curve for malignancy grade (MG $-)$ and $5 \mathrm{c}$ exceeding rate $(5 \mathrm{cER} ;---)$. Area under the curve for MG $=0.87(0.776-$ 0.965). The value of MG represented at the point on the curve closest to the upper left-hand corner was 0.10 . At this level its sensitivity and specificity were 80 and 93.6\%, respectively. Area under the curve for $5 c E R=0.812(0.699-0.925)$.

\begin{tabular}{|c|c|c|c|c|c|}
\hline \multirow[t]{2}{*}{$\begin{array}{l}\text { Histological type/ } \\
\text { clinical stage }\end{array}$} & \multirow[t]{2}{*}{ Total } & \multicolumn{2}{|c|}{$\begin{array}{l}\text { DNA cytometric } \\
\text { results }\end{array}$} & \multicolumn{2}{|c|}{$\begin{array}{l}\text { Cytology } \\
\text { results }\end{array}$} \\
\hline & & $\begin{array}{c}\text { Grade } \\
\text { II }\end{array}$ & $\underset{1}{\text { Grade }}$ & $\begin{array}{l}\text { High } \\
\text { grade }\end{array}$ & $\begin{array}{c}\text { Normal/ } \\
\text { low grade }\end{array}$ \\
\hline Squamous cell & 9 & 9 & 0 & 1 & 8 \\
\hline Severe dysplasia & 2 & 2 & 0 & 0 & 2 \\
\hline Carcinoma in situ & 2 & 2 & 0 & 1 & 1 \\
\hline Cancer stage I & 2 & 2 & 0 & 0 & 2 \\
\hline Cancer stage II & 2 & 2 & 0 & 0 & 2 \\
\hline Cancer stage $>$ II & 1 & 1 & 0 & 0 & 1 \\
\hline Adenocarcinoma & 11 & 8 & 3 & 0 & 10 \\
\hline Cancer stage I & 4 & 1 & 3 & 0 & 3 \\
\hline Cancer stage ॥ & 1 & 1 & 0 & 0 & 1 \\
\hline Cancer stage $>\| 1$ & 6 & 6 & 0 & & 6 \\
\hline Small cell & 1 & 0 & 1 & 0 & 1 \\
\hline Others & 4 & 3 & 1 & 0 & 1 \\
\hline
\end{tabular}

one metastasis of a nonlung cancer and two without pathological type characterisation. Among these cases, only one without a pathological result had a negative result on image cytometry.

\section{DISCUSSION}

Lung cancer has been described as an epidemic disease with high mortality, spreading from males to females, and from the old to the young. The high-risk population is vast and growing [23]. It has been well demonstrated that patients with early stage lesions have a much better prognosis than those in advanced stage. However, until now, there have been no accepted screening strategies to improve the early detection rate of lung cancer [23]. Recently, population-based trials of CT screening were launched in several countries to assess the utility of CT in the early detection of lung cancer [7]. To the present authors' knowledge, no data based on population studies have been reported concerning the assessment of utility of sputum examination using modern technology. Evaluating the efficiency of automated sputum image cytometry and fluorescence bronchoscopy in the detection of early lung cancer was one of the principle aims of the RIDTELC Lung Study. Several investigators have reported the role of $5 \mathrm{cER}$ and $2 \mathrm{cDI}$ as well as MG, in identifying malignancy in several kinds of cancer, e.g. breast, prostate and cervix uteri malignant tumours [21]. In this study the percentage of aneuploid cells with a DNA content above 5cER and 2cDI, measured by automated sputum image cytometry, were employed as markers for malignant or pre-malignant cells in sputum, to determine the presence of malignant lesions in the lung. MG computed from both markers was used as a decision parameter. Results shown in table 1 suggest that the population with suspicious automated sputum cytology results were characterised by higher inhalation of tobacco, simultaneously occupational exposure to harmful agents and more complaints of a chronic cough; all of which are in accordance with the results of epidemiological studies assessing risk factors in lung cancer $[16,24]$. This may well indicate that those individuals with positive cytometric results may be considered as a particularly high-risk population for the development of lung cancer in heavy smokers. Follow-up will be needed to confirm this observation.

In the current study, the prevalence of lung cancer was $1.1 \%$ (27 out of 2,480), which is similar to the expected prevalence of $1-2 \%$. Of the 27 patients found with cancer during the study, two did not receive automated sputum cytology screening. Of the remaining 25, only 11 had positive results by sputum examination, one had both cytology and cytometry positive results, and 10 had only cytometry positive results. The sensitivity could be improved from 4.2 to $80 \%$, but positive predictive values were similar (10 versus 11.4\%) if automated sputum cytometry was used instead of conventional cytology (table 2). Severe dysplasia or higher lesions were considered to be positive results by conventional sputum cytology examination. Table 4 shows all nine patients with squamous cell cancer had positive DNA cytometric results, demonstrating a $100 \%$ sensitivity of image cytometry for this type of lung cancer. Most importantly, eight out of nine patients were found at early stage (0-II) and only one was at a later stage (III). Therefore, squamous cell carcinoma of the lung could be detected at an early stage by DNA image cytometry. For adenocarcinoma of the lung, a high detection rate was also evident: eight out of 11 patients $(72.7 \%)$ had positive DNA cytometric results, nevertheless, only two out of eight patients detected were at early stage (I-II). Therefore, the current data show that the sensitivity of image cytometry was much higher than that of conventional cytology in predicting the presence of clinically relevant lung cancer, especially the early stage squamous cell cancer, $80 \%$ against $4.2 \%(\mathrm{p}<0.001)$. If the early detection rate by sputum image cytometry and conventional 
sputum cytology are compared, it could be found that among 13 early stage cancers ( 0 -II stage), 10 could be detected by cytometry, but only one could be detected by sputum cytology. As a result, it could be expected that more cancers, especially more early stage cancers, may be detected if the high-risk population is primarily screened by sputum cytometry rather than by conventional cytology examination. Nevertheless, there is no way to know how many asymptomatic patients have negative automated sputum cytology and cytology results in the current study, which is one limitation. Hence, the accurate specificity and negative predictive value of automated sputum cytometry could not be predicted from the present data. It is worthy to note that the procedure of bronchoscopy for diagnosis of lung cancer has a high reliability, as shown in table 3. So far, no false-negative findings occurred, all biopsies from bronchoscopical normal areas had no high-grade histopathological results. A high prevalence of low-grade lesions was revealed by pathological examination in $43.2 \%$ of the subjects having grade II results by sputum cytometry.

SACCOMANNO et al. [25] demonstrated that in heavy smokers it took about 9.2 yrs from the first detection of mild dysplasias in sputum to invasive cancer. Furthermore, only a portion of preinvasive lesions develop into invasive lung cancer [26, 27]. Conventional sputum cytology failed to prospectively identify the progression to malignancy in the patients with pre-invasive lesions. Presently, there is no method capable of doing this. Sputum image cytometry could have potential in this respect. As shown in figure 1, 5cER was significantly different among groups with histologically confirmed normal, pre-invasive, and malignant lesions. It was increased with histological progression from normal through pre-invasive to malignant lesions, with median values of $0.034,0.051$, and $0.070 \%$, respectively, which suggested that the high level of $5 \mathrm{cER}$ was related to the respective stage of development of pulmonary malignancy. Similar results have also been reported in other cancer studies $[15,28]$. It has been reported that $10-30 \%$ of bronchial carcinomas develop without cytomorphologically diagnostic malignant cells [29]. Morphological abnormalities of cancer cells may be reflected by DNA aneuploidy more than by their proliferative activity in nonsmall cell lung cancer [30]. This was confirmed by the current results, showing a lower level of 5 cER in the group without histological abnormalities. Taken together, the current results suggest that observation of 5 cER may be valuable in predicting the progression of pre-invasive tissues. No significant relationship between histological changes in the transition from normal through pre-invasive to invasive stage, and the values of $2 \mathrm{cDI}$ and the combined parameter MG were found in this study. Nevertheless using $5 \mathrm{cER}$ alone to predict the presence of lung cancer has its limitations, because not all lung cancers contain aneuploidy. A meta-analysis of 35 related studies [31] set the prevalence of aneuploidy in nonsmall cell lung cancer at 64$67 \%$. MG may overcome this drawback, therefore the current authors used it as a decisive parameter to make a cytometric diagnosis in this study. To compare predictive values of the two parameters, a ROC curve was drawn, the area under the ROC curve was 0.87 for MG and 0.81 for $5 \mathrm{cER}$, presenting the diagnostic accuracy as $87 \%$ for MG against $81.2 \%$ for $5 \mathrm{cER}$. It is obvious that diagnostic accuracy of automated sputum image cytometry could be decreased using $5 \mathrm{cER}$ instead of MG as the decision parameter. The consideration of combining these two parameters to predict the presence and occurrence of a malignant tumour is valuable. It was striking that in 66 out of $95(69 \%)$ subjects with suspicious cytometric results, no bronchoscopical abnormality could be localised. This has also been observed in individuals with DNA abnormalities in cells of the oral cavity and oesophagus [32].

This evaluation was based solely on DNA content abnormality. Image cytometry is a unique method allowing the quantitative description of the textural parameters of a stained nuclear structure in a cell. It can measure a great number of features of nuclei, which are linked to malignancy. Several studies have demonstrated that malignancy associated changes, measured by image cytometry, also have a higher diagnostic accuracy for diagnosis of lung cancer. In a field study, features such as ploidy, mean nuclear radius, optical density variance, optical density skew and average range of optical density were used as parameters to discriminate malignant from benign lesions [33]. In this study, the sensitivity of automated image cytometry was higher for adenocarcinoma than squamous cell lung cancer.

Automating of image cytometry enhances its efficiency in screening slides, thereby allowing 50 slides to be automatically processed per day. The present software version permits up to 50,000 objects (cells) to be identified and analysed in $30 \mathrm{~min}$. Applied to primary screening, this method has a low level of personnel costs. Thus, DNA image cytometry appears to be a robust and economic tool for lung cancer screening.

In conclusion, DNA image cytometry might be a suitable tool for identifying a population with a particularly high risk of developing lung cancer, e.g. in heavy smokers. $5 c$ exceeding rate could serve as a parameter to monitor the progression of a pre-invasive lesion. Importantly, malignancy grade, combining $5 c$ exceeding rate and $2 c$ deviation index measurements was a useful parameter for early detection of lung cancer by automated sputum image cytometry, especially for squamous cell lung carcinoma. For the early diagnosis of adenocarcinoma of the lung, other parameters should be investigated in order to increase the diagnostic sensitivity of image cytometry. Automated sputum image cytometry appears a feasible screening tool to detect lung cancer at an early, asymptomatic stage. However, its specificity should be further investigated.

\section{REFERENCES}

1 Parkin DM, Pisani P, Ferlay J. Global cancer statistics. $C A$ Cancer J Clin 1999; 49: 33-64.

2 Fry WA, Menck HR, Winchester DP. The national cancer data base report on lung cancer. Cancer 1996; 77: 19471955.

3 Mountain CF. Revisions in the international system for staging lung cancer. Chest 1997; 111: 1710-1717.

4 Melamed MR, Flehinger BJ, Zaman MB, Heelan RT, Perchick WB, Martini N. Screening for early lung cancer: results of the Memorial Sloan-Kettering Study in New York. Chest 1984; 86: 44-53.

5 Frost J, Ball WC, Levin JL, et al. Early lung cancer detection: results of the initial (prevalence) radiologic and cytologic 
screening in the Johns Hopkins Study. Am Rev Respir Dis 1984; 130: 549-554.

6 Fontana RS, Sanderson DR, Woolner LB, Taylor WF, Miller WE, Muhm JR. Lung cancer screening: the Mayo program. J Occup Med 1986; 28: 746-750.

7 Field JK, Brambilla C, Caporaso N, et al. Consensus statements from the second international lung cancer molecular biomarkers workgroup: a European strategy for developing lung cancer diagnostics in high risk populations. Int J Oncol 2002; 21: 369-373.

8 Hirsch FR, Franklin W, Gazdar AF, Bunn PA. Early detection of lung cancer: clinical perspectives of recent advances in biology and radiology. Clin Cancer Res 2001; 7: 5-22.

9 Marek W, Kotschy-Lang N, Muti A, et al. Can semiautomated image cytometry on induced sputum become a screening tool for lung cancer? Eur Respir J 2001; 18: 942-950.

10 Haroske G, Baak JP, Danielsen HE, et al. Fourth updated ESACP consensus report on diagnostic DNA image cytometry. Anal Cell Pathol 2001; 23: 89-95.

11 Duesberg PH, Rasnick D. Aneuploidy, the somatic mutation that makes cancers a species of its own. Cell Motil Cytoskeleton 2000; 47: 81-107.

12 Yamamoto T, Horiguchi H, Kamma K, et al. Comparative DNA analysis by ICM and FCM in non-small cell lung cancer. Jpn J Cancer Res 1994; 85: 1177.

13 Planz B, Synek C, Robben J, et al. Diagnostic accuracy of DNA image cytometry and urinary cytology with cells from voided urine in the detection of bladder cancer. Adult Urology 2000; 56: 782-786.

14 Chatelain R, Schunck T, Schindler E, et al. Diagnosis of prospective malignancy in koliocytic dysplasias of the cervix with DNA cytometry. J Reprod Med 1989; 34: 505-510.

15 Remmersbach $\mathrm{T}$, Weidenbach $\mathrm{H}$, Pomjanski $\mathrm{N}$, et al. Cytologic and DNA-cytometric early diagnosis of oral cancer. Anal Cell Pathol 2001; 22: 221.

16 Jöckel KH, Ahrens W, Jahn I, Pohlabeln H, BolmAudorff U. Occupational risk factors for lung cancer: a case-control study in West-Germany. Int J Epidemiology 1998; 27: 549-560.

17 Papanicolaou GN. A new procedure for staining vaginal smears. Science 1942; 95: 438-439.

18 Schulte E, Wittekind DH. Standarization of the Feulgen reaction: the influence of chromatin condensation on the kinetics of acid hydrolysis. Anal Cell Pathol 1990; 2: 149-157.
19 Papanicolaou GN. Atlas of Exfoliative Cytology. Cambridge, MA, Harvard University Press, 1954.

20 Böcking A, Giroud F, Reith A. Consensus report of the ESACP task force on standardization of diagnostic DNA image cytometry. Anal Cell Pathol 1995; 8: 67-74.

21 Böcking A, Hilgarth M, Auffermann W, Hack-Werdier C, Fischer-Becker D, von Kalkreuth G. DNA-cytometric diagnosis of prospective malignancy in borderline lesions of the uterine cervix. Acta Cytol 1986; 30: 608-614.

22 Delong ER, Delong DM, Clarke-Pearson DL. Comparing the areas under two or more correlated receiver characteristic curves: a nonparametric approach. Biometrics 1988; 44: 837-845.

23 Mulshine JL. Screening for lung cancer: in pursuit of premetastatic disease. Nat Rev Cancer 2003; 3: 65-73.

24 Ruano-Ravina A, Figueiras A, Barreiro-Carracedo MA, et al. Occupation and smoking as risk factors for lung cancer. Am J Ind Med 2003; 43: 149-155.

25 Saccomanno G, Archer VE, Auerbach O, Saunders RP, Brennan LM. Development of carcinoma of the lung as reflected in exfoliated cells. Cancer 1974; 33: 256-270.

26 Bota S, Auliac J-B, Paris C, Métayer J, Sesboüé R. Follow-up of bronchial precancerous lesions and carcinoma in situ using fluorescence endoscopy. Am J Respir Crit Care Med 2002; 164: 1688-1693.

27 Venmans BJW, van Boxem TJM, Smit EF, Postmus PE, Sutedja TG. Outcome of bronchial carcinoma in situ. Chest 2000; 117: 1572-1576.

28 Inage $\mathrm{Y}$, Ogata $\mathrm{T}$, Yamamoto $\mathrm{E}$, et al. Topographical analysis of p53 expression and DNA ploidy in early bronchial squamous cell carcinoma and preneoplastic lesions. Lung Cancer 2001; 34: 351-361.

29 Auer G, Ono J, Nasiell M, Casperson T, Kato H, Konaka C. Reversibility of bronchial cell atypia. Cancer Res 1982; 42: 4241-4247.

30 Yamamoto T, Noro M, Hiroguchi H. Nuclear abnormalities of cancer cells in morphology are greatly influenced by DNA ploidy but not by proliferative activity in non-small cell lung cancer (NSCLC). Lung Cancer 1997; 18: 178.

31 Choma D, Daures JP, Quantin X, et al. Aneuploidy and prognosis of non-small cell lung cancer: a meta-analysis of published data. Br J Cancer 2001; 85: 14.

32 Sudbo J, Ried T, Bryne M, et al. Abnormal DNA content predicts the occurrence of carcinomas in non-dysplastic white patches. Oral Oncology 2004; 37: 558-565.

33 Palcic B, Garner D, Beveridge J, et al. Increase in sensitivity of sputum cytology using high-resolution image cytometry: field study results. Cytometry 2002; 50: 168-176. 